\title{
Clinical Presentation and Treatment Outcome of Abdominal Tuberculosis
}

\author{
OLIURRAHMAN, ${ }^{1}$ ANWARUL KABIR, ${ }^{2}$ PRODIP KUMAR BISWAS, ${ }^{3}{\text { AMM SHORIFUL ISLAM, }{ }^{4} \text { ABSIDDIK, }{ }^{5} \text { HABIBUR RAHMAN }}^{6}$
}

\begin{abstract}
:
Background: Tuberculosis is common in our country. It commonly affects the lungs but in up to one third of the cases involve other organs. Abdominal tuberculosis is one of the important extrapulmonary tuberculosis.lt has varied presentation, frequently mimicking other common and rare diseases.
\end{abstract}

Materials and Methods:It is a prospective observational study done to see the clinical presentation and treatment outcome of abdominal tuberculosis in medicine, surgery and gastroenterology department Sylhet M A G Osmani Medical College Hospital during Jan 2008-Dec2008. Sample was collected purposively. All patients were undergone detailed history, clinical examination, relevant investigation and follow up till the end of six months anti tubercular chemotherapy.Data recorded in predesigned record form. Statistical analysis was done with SPSS software. All data presented as percentage or mean.

Result: From the 50 cases of abdominal TB, 26 (52\%) were male and 24 (48\%) were female. Male and female ratio was I.04:I. Here most of the patients were in the age of $2 \mathrm{I}-30$ years and mean age was 29.3 years, majority of them were from poor socioeconomic status $(18 \%)$ had associated pulmonary tuberculosis. The common presentation exhibited by the patients were weight loss (100\%), low grade fever (88\%), abdominal pain (86\%), bowel disturbance (84\%), anorexia (80\%), nausea and vomiting (70\%), abdominal distension (46\%), , ascites (42\%).Most of the patients were anaemic with raised ESR.Ultrasonography revealed ascites,abdominal lymphadenopathy and thick walled bowel. Colonoscopy found nodularity and ulceration with narrowing. Ascitic fluid was exudative with predominant lymphocyte count. Laparoscopy and laparotomy revealed tubercle on the intestine and the mesentery with thickening and bowel stricture. All the biopsy reports were consistent with tuberculosis. Site of involvement in decreasing frequency were ileocaecal (44\%), peritoneal (I6\%), abdominal lymph node (8\%), ascending colon (8\%), small bowel $(6 \%)$,transverse colon (4\%), sigmoid colon (2\%), rectum (2\%), gastric (2\%) and duodenal (2\%) but multiple site in $6 \%$. The treatment was given as per the WHO guidelines with category I drugs and found- $86 \%$ cured with anti TB alone, $4 \%$ needed surgery for intestinal obstruction along with anti TB, and 10\% drop out from follow up.

Conclusion:The clinical presentations of abdominal TB appear not specific for the condition. Thus, careful approach and supportive results are required in order to issue the final diagnosis. If diagnosed early, it can be treated successfully with the conventional anti-TB drugs.

Key words: Abdominal tuberculosis(TB), treatment outcome, antitubercular chemotherapy (ATT)

\section{Introduction:}

Tuberculosis is an infectious disease, caused by bacilli called Mycobacterium tuberculosis complex. Abdominal tuberculosis can involve the luminal gastrointestinal tract, liver, spleen, peritoneum and female genital tract. ${ }^{1}$ Worldwide, one person out of three is infected with Mycobacterium tuberculosis - two billion people in total. The disease usually affects the lungs although in up to one third of cases other organs are involved. ${ }^{2}$ It can involve any part of the

1. Asst. Professor of Gastroenterology, SoMC

2. Professor of Gastroenterology, BSMMU

3. Resident, Gastroenterology, BSMMU

4. Asst. Professor of Gastroenterology, DMC

5. RMO, Fatulla 20 Beded Hospital, Narayanganj

6. Professor of Gastroenterology, SSMC

Correspondence : Dr. Oliur Rahman, Asst. Professor of Gastroenterology, SoMC. E-mail: oli.dmc53@yahoo.com. gastrointestinal tract from mouth to anus, the peritoneum and the pancreatobiliary system. It can have a varied presentation, frequently mimicking other common and rare diseases. ${ }^{3}$ Abdominal tuberculosis is predominantly a disease of the young adult. ${ }^{4}$ The most common site of involvement is the ileocaecal region. The classic histological finding is a caseating granuloma. In tuberculous peritonitis, it is studded with multiple yellow white tubercles. The clinical presentation may be acute or chronic or acute on chronic. Even patient may be without symptoms. Most patients have constitutional symptoms of fever (40-70\%), pain (80-95\%), diarrhoea (11-20\%), constipation, alternating constipation and diarrhoea, weight loss (40-90\%), anorexia and malaise. Pain can be either colicky or dull \&continuous. An abdominal mass may be palpable. Many patients have no pulmonary symptoms. The study was carried to see the clinical presentation and treatment outcome of abdominal 
tuberculosis, which will be helpful in diagnosis and management of abdominal tuberculosis. Few works have been done previously on abdominal tuberculosis but number of patient was less than the present study and no follow up study of treatment outcome was done.

\section{Materials and Methods:}

All consecutive cases diagnosed as abdominal tuberculosis fulfilling the exclusion and inclusion criteria are included in this study. Fifty cases of both male and female patient of 15 years and above admitted in different medical and surgical units of Sylhet M.A.G. Osmani Medical College Hospital from January' 08 to December'08 were included in this study. Patients were selected on the basis of clinical presentations and investigation including histopathology. Abdominal tuberculosis is diagnosed by histopathological evidence of caseating granuloma. Investigation such as Stool R/E, blood for TC, DC, ESR, $\mathrm{Hb} \%$, S.bilirubin, SGPT, prothrombin time, Alkaline phosphatase, S. Creatinine, RBS/FBS, MT, CXR, Ultrasonography of whole abdomen were done for all the patients. For those patients with ascites, ascitic fluid study including biochemical and cytological examination and AFB staining and if needed peritoneal biopsy was done. Endoscopy of upper GIT, full length colonoscopy and biopsy for histopathology had been done for patient with ascites \&/or alteration of bowel habit. If histopathology was negative but clinical features are suggestive then diagnostic Laparoscopy \&/or Laparotomy \& biopsy were performed. Contrast radiography in the form of barium follow through was done if needed. For patient with palpable abdominal lump and/or abdominal lymphadenopathy, USG/CT guided FNAC had been done. Those patients having pulmonary lesions suggestive of tuberculosis on chest x-ray, sputum for AFB staining was done. Category I antitubercular therapy was given for each patient.Complication of antitubercular chemotherapy and necessary measures if any complication developed were explained. Follow up of the patient were done monthly for the first two month \& thereafter two monthly up to sixth months. They were assessed clinically and from laboratory support for $\mathrm{Hb} \%$ and ESR. This study had included the response of 6 months anti tubercular regimen.

\section{Diagnostic algorithm of abdominal tuberculosis}

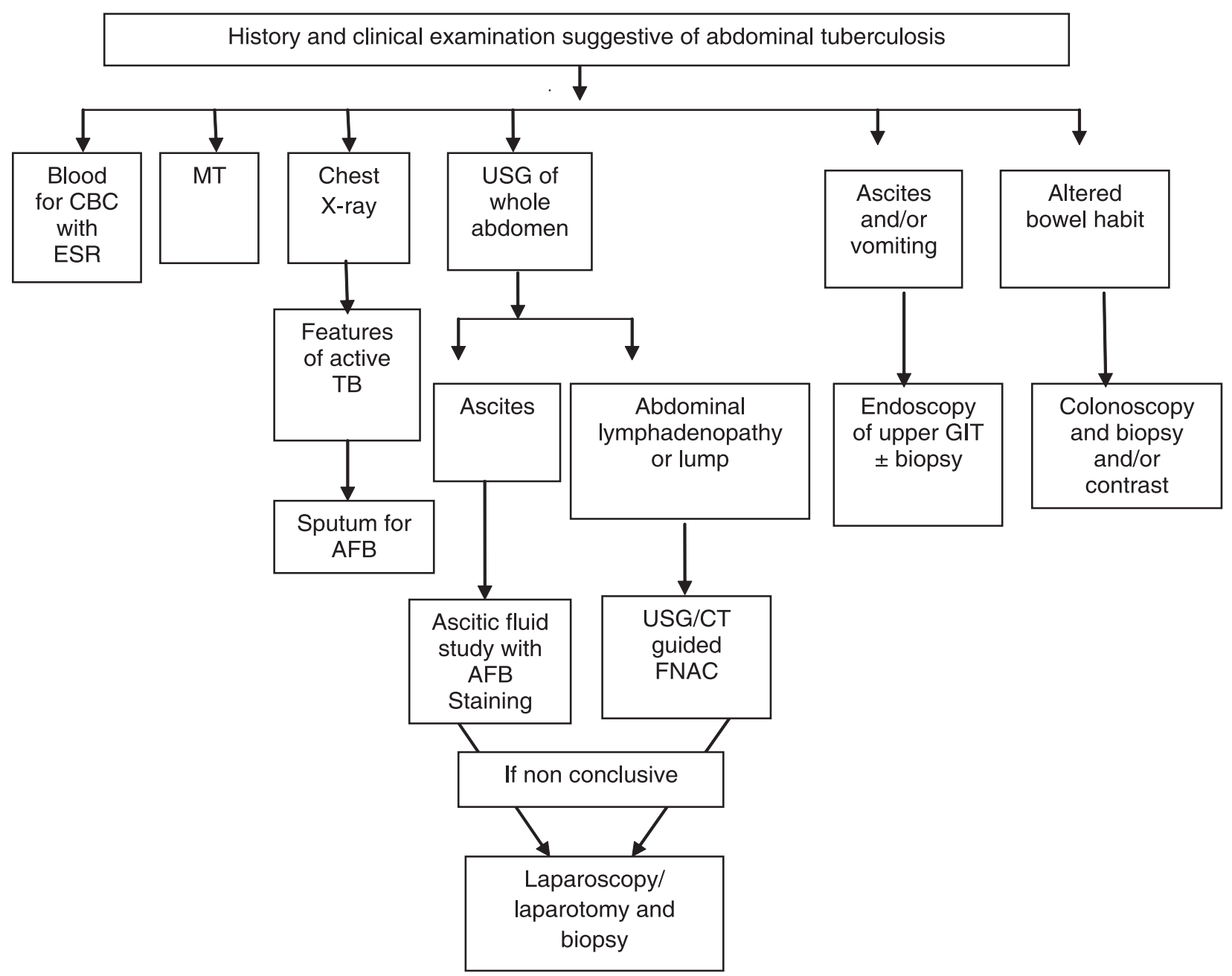




\section{Results:}

During the period of January, 2008 to December, 2008 , 50 patients were observed with Male and female ratio was 1.04:1, most of the patients were in the age of 21-30 years and mean age was 29.3 years, $68 \%$ were poor socioeconomic group, $84 \%$ rural dweller, Duration of presentation varies from 1 month $-2 \frac{1}{2}$ years. But most of the patients presented within 1 year of symptoms, $42 \%$ had contact with pulmonary tuberculosis patient, $18 \%$ had associated pulmonary tuberculosis, $8 \%$ had past history of tuberculosis. Common presentations were weight loss $(100 \%)$, low grade fever $(88 \%)$, abdominal pain $(86 \%)$, bowel disturbance $(84 \%)$, anorexia $(80 \%)$, nausea and vomiting (70\%), abdominal distension $(46 \%)$, ascites $(42 \%)$. Site of involvement in decreasing frequency were ileocaecal (44\%), peritoneal (16\%), abdominal lymph node (8\%), ascending colon (8\%), small bowel (6\%), transverse colon $(4 \%)$, sigmoid colon $(2 \%)$, rectum $(2 \%)$, gastric ( $2 \%)$ and duodenal $(2 \%)$ but multiple site in $6 \%$.

Table I

$\mathrm{Hb} \%$ and ESR in patients of abdominal TB

\begin{tabular}{lcc}
\hline Features & Mean & Range \\
\hline $\mathrm{Hb} \%$ & $8.5 \mathrm{gm} / \mathrm{dl}$ & $6-11 \mathrm{gm} / \mathrm{dl}$ \\
ESR & $55 \mathrm{~mm} / \mathrm{h}$ & $25-110 \mathrm{~mm} / \mathrm{h}$ \\
\hline
\end{tabular}

Table II

MT result in patients of abdominal TB(Induration $>10$ $\mathrm{mm}$ is regarded as positive.)

\begin{tabular}{lcc}
\hline Result & Number of patients $(\mathrm{n}=50)$ & Percentage $(\%)$ \\
\hline Positive & 30 & 60 \\
Negative & 20 & 40 \\
\hline
\end{tabular}

Table III

Treatment outcome of abdominal TB

\begin{tabular}{lcc}
\hline Features & $\begin{array}{c}\text { Number of patients } \\
(\mathrm{n}=50)\end{array}$ & $\begin{array}{c}\text { Percentage } \\
(\%)\end{array}$ \\
\hline Cured & 43 & $86 \%$ \\
Cured but needed surgery & 2 & $4 \%$ \\
with ATT & & $10 \%$ \\
Drop out from follow up & 5 & \\
\hline
\end{tabular}

\section{Discussion:}

TB of the gastrointestinal tract is the sixth most frequent form of extra-pulmonary site..$^{5}$ It has varied presentation, frequently mimicking other common and rare diseases. This study reports the socio-demographic factors, clinical presentation and treatment outcome which would help the physician to diagnose and treat this curable disease. From the 50 patients, $26(52 \%)$ were male and $24(48 \%)$ were female. Male and female ratio was 1.04:1. Tariq $\operatorname{Sinan}^{6}$ et al also showed male predominance in his study. Here most of the patients were in the age of 21-30 years and average age was 29.3 years, which is similar to Prakashet al. ${ }^{7}$ A previous study from Bangladesh also reported similar type of result. It is also known that two thirds of the patients with abdominal TB are 21-40 years old. ${ }^{8}$ In our study we found such an observation. Majority of the patients $(68 \%)$ were in poor socio-economic condition and $84 \%$ of them were rural dweller. Similar results were shown by Vaidyaet $\mathrm{al}^{9}$ and one of the study in our country. Forty two percent of the patients had positive history of contact but $25 \%$ was shown by Prakashet al. $^{7} 8 \%$ of cases had past history of pulmonary tuberculosis, $18 \%$ of the patients had associated pulmonary tuberculosis, but Muneef MA et $\mathrm{al}^{10}$ found $30 \%$ of the patients either gave past history of TB or presented with active TB of other sites. Duration of presentation varies from 1 month $-2 \frac{1}{2}$ years, but average duration was 6 months. In this series $8 \%$ of the patients presented with intestinal obstruction and $4 \%$ with intestinal perforation, but Khan Ret $\mathrm{al}^{11}$ found acute intestinal obstruction was seen in $11 \%$. Common presentation were low grade fever (88\%), abdominal pain (86\%), weight loss $(100 \%)$, anorexia (80\%), nausea and vomiting $(70 \%)$, abdominal distension (46\%), bowel disturbance (84\%), ascites $(42 \%)$ which were similar toP.V. Kishore et al. ${ }^{12}$ Abdominal lump and abdominal lymphadenopathy were $28 \%$ and $12 \%$ respectively whereas Uygur-Bayramicli $\mathrm{O}$ et a ${ }^{13}$ found $16.8 \%$ tuberculous lymphadenitis and Muneef MA et a ${ }^{10}$ showed abdominal mass $13 \%$. Site of involvement in decreasing frequency were ileocaecal (44\%), peritoneal $(16 \%)$, abdominal lymphnode $(8 \%)$, which were similar toKhan Ret $\mathrm{al}^{11}$. In most of the study, intestinal TB is commonest and ileocaecal $(21-24)^{9}$ is the predominant site of involvement. In this seriesaverage $\mathrm{Hb} \%$ was $8.5 \mathrm{gm} / \mathrm{dl}$ which were similar to Prem Mukerjee et a ${ }^{14}$ and Uzunkoy A et al. ${ }^{15}$ Sputum for AFB was positive in $6 \%$ of cases, chest $\mathrm{x}$ ray showed abnormality in $18 \%$ cases, Akinkuolie AA et al ${ }^{16}$ found Chest X-ray showed abnormal findings in $25 \%$ of the patients and sputum was positive for AFB in $14.3 \%$. USG revealed abnormality in $60 \%$ of cases and predominant findings were ascites in $42 \%$ cases, lymphadenopathy in $12 \%$ cases which is similar to Uygur-Bayramicli $\mathrm{O}$ et al. ${ }^{13}$ Colonoscopy revealed- nodularity and ulceration in $84 \%$, ulcer in $9 \%$ and narrowing in $6 \%$ of cases which is alike toUygur-Bayramicli O et al. ${ }^{13}$ So ulcerative lesion is common in intestinal TB.Ascitic fluid study revealed exudative fluid with lymphocyte predominance but no AFB was found which 
were like toUygur-Bayramicli Oet al. ${ }^{13}$

Fine needle aspiration cytology and biopsyreports were consistent with tuberculosis in $100 \%$ cases which were similar to Bhargavaet al. ${ }^{17}$ So it is a very specific investigation when there is diagnostic dilemma. Laparotomy was done in 4 cases2 cases were intestinal perforation, 2 were intestinal obstruction and histopathological reports were consistent with tuberculosis in $100 \%$ cases which were like toVKS Leung et al. ${ }^{18}$ The management of abdominal TB requires conventional anti TB for at least 6 months including initial 2 months of rifampicin, INH, Pyrazinamide and Ethambutol. In our study though all the patients were treated with 6 months regimen and found- $86 \%$ cured with anti TB alone, $4 \%$ needed surgery for intestinal obstruction along with anti $\mathrm{TB}$, and $10 \%$ drop out from follow up.

\section{Conclusion:}

Abdominal tuberculosis has diverse and nonspecific symptomatology.Thus, careful approach and supportive results are required in order to issue the final diagnosis. If diagnosed early, it can be treated successfully with the conventional anti-TB drugs.

\section{Conflict of Interest: None}

\section{References:}

1. Tya-Mae Y. Julien MD. Current Diagnosis and Treatment in Gastroenterology, $2^{\text {nd }}$ ed. McGraw-Hill.2003; 10: 166-76.

2. Mario C. Raviglione, Richard J. O’ Brien, Tuberculosis- In kasper DL, Fauci AS, Logo DL, Braun Wald E, Hausers, Jameson JL, editors Harrisons, Principles of Internal Medicine, New York, McGraw-Hill 16th ed. 2005; 1: 95359.

3. MP Sharma, Bhatia V. Abdominal tuberculosis. Indian J. Med. 2004; 120(4): 305-15.

4. Niall O Aston MA,Chir M. Abdominal tuberculosis. World Journal of Surgery 1997; 21: 492-99.

5. Daniel TM. The history of tuberculosis.Respir Med 2006; 100: $1862-70$.

6. Sinan T, Sheikh M, Ramadan S, Sahwney S, Behbehani A. CT features in abdominal tuberculosis: 20 years experience, BMC Medical Imaging 2002; 2:3.
7. Prakas, A.Ulceroconstrictivetuberculosis of the bowel. International surgery, 1978; 63: 23-29.

8. Bhargava DK, Shriniwas, Chopra P, Nijhawan S, Dasarathy S, Kushwaha AK. Peritoneal tuberculosis: laparoscopic patterns and its diagnostic accuracy. Am J Gastroenterol 1992; 87: 109-12.

9. Vaidya MG, SodhiJS. Gastrointestinal tract tuberculosis; A study of 102 cases including 55 hemicolectomies Clinical radiology, 1978; 29: 189-95.

10. Muneef M A, Memish Z, Mahmoud SA, Sadoon SA, Bannatyne R, Khan Y. Tuberculosis in the belly: a review of forty-six cases involving the gastrointestinal tract and peritoneum.Scand J Gastroenterol 2001; 36(5): 528-32.

11. Khan AR, Abid S, Jafri W, Abbas Z, Hameed K, Ahmad Z, Diagnostic dilemma of abdominal tuberculosis in non-HIV patients: an ongoing challenge for physicians, World J Gastroenterol 2006; 12(39): 6371-5.

12. Kishore PV, ChandrsekharTS, Palaian S. Diagnosing Abdominal Tuberculosis: A Retrospective Study From Nepal. The Internet Journal of Gastroenterology 2008; 6(2): 87-89.

13. Bayramicli O, Dabak G, Dabak R. A clinical dilemma: abdominal tuberculosis. World J Gastroenterol 2003; 9(5): 1098-101.

14. Mukerjee P, Rajor R.Abdominal Tuberculosis. Ind. J. Tub; 26 (2): 62-66.

15. Uzunkoy A, Harma M, Harma M, Diagnosis of abdominal tuberculosis: experience from 11 cases and review of the literature, World J Gastroenterol. 2005; 11(21): 3328.

16. Akinkuolie AA, Adis AO, AqbakwaruEA, Eqharevba, PA, Adesunkanmi AR, Abdominal tuberculosis in a Nigerian teaching hospital, Afr J Med Med Sci. 2008; 37(3): 225-9.

17. Bhargava DK, Shriniwas, Chopra P, Nijhawan S, Dasarathy S, Kushwaha AK. Peritoneal tuberculosis: laparoscopic patterns and its diagnostic accuracy. Am J Gastroenterol 1992; 87: 109-12.

18. LeungVK, Law ST, Lam CW, LukI SC, Loke TKL, Chan WH, Lam SH. Intestinal tuberculosis in a regional hospital in Hong Kong: a 10- year experience. Hong Kong Med J. 2006; 12(4): 264-71. 